\title{
Entre attaches régionales et service de l'État : les inspecteurs primaires de l'Ouest au XIXe siècle
}

\section{Gilbert Nicolas}

\section{(C) OpenEdition}

1 Journals

\section{Édition électronique}

URL : http://journals.openedition.org/abpo/1552

DOI : $10.4000 / a b p o .1552$

ISBN : 978-2-7535-1487-4

ISSN : 2108-6443

Éditeur

Presses universitaires de Rennes

Édition imprimée

Date de publication : 20 septembre 2002

Pagination : 33-56

ISBN : 978-2-86847-768-2

ISSN : 0399-0826

\section{Référence électronique}

Gilbert Nicolas, «Entre attaches régionales et service de l'État: les inspecteurs primaires de l'Ouest au

XIXe siècle », Annales de Bretagne et des Pays de l'Ouest [En ligne], 109-3 | 2002, mis en ligne le 20 septembre 2004, consulté le 27 avril 2019. URL : http://journals.openedition.org/abpo/1552 ; DOI : 10.4000/abpo. 1552 


\title{
Entre attaches régionales et service de l'État: les inspecteurs primaires de l'Ouest au XIX siècle
}

\author{
Gilbert NICOLAS \\ Maître de conférences en histoire contemporaine, \\ CRHISCO-Université de Rennes 2
}

Depuis la Révolution française, trois forces se disputent le contrôle de l'école primaire en France : l'Église, les notables et l'État ${ }^{1}$. Au cours du $\mathrm{XIX}^{\mathrm{e}}$ siècle, précisément à partir de 1835, la mise sur pied d'un corps d'inspection primaire permanent, déjà étudié dans le cadre national par Christian Roux et Jean Ferrier, incarne la reprise en main progressive de l'école primaire par l'État, avec le double objectif de la connaissance et de la surveillance des écoles.

Ce regard et ce contrôle, délégués par l'État au corps d'inspection, se développent progressivement sous quatre régimes successifs, malgré la concurrence d'autres surveillants locaux, malgré les multiples résistances, malgré une redéfinition de la géographie administrative de l'Instruction publique, en particulier en 1850 et en 1854, et une évolution par à-coups de l'action de l'État, fortement tributaire des changements de pouvoir politique $^{2}$ et des nouvelles législations (1830-1833, 1848-1850, 1852, 1854, 1870, 1877-1879).

La présente recherche porte d'abord sur la mise en place et le fonctionnement du corps de l'inspection au sein d'une grande académie de l'Ouest français, considérée comme " délicate ${ }^{3}$ " par tous les régimes en place, de la monarchie de Juillet à la Troisième République, disons de 1835

1. Roux, Christian, L'inspection primaire et la surveillance de l'école en France au XIXe siècle, Doctorat d'État, Aix-Marseille 1, 1994, p. 25.

2 . Nous n'écrivons pas " changements de régimes politiques " car l'expression est trop restreinte; dans la première décennie de la Troisième République, par exemple, les changements de gouvernements ont des répercussions sur la carrière des inspecteurs, en particulier dans la période 1877-1879.

3. AN F17/2649, microfilm : rapport trimestriel politique du recteur de l'académie de Rennes au ministre, 7 janvier 1859. Cet adjectif est emprunté au texte du recteur de l'académie de Rennes, Mourier. Ce dernier est recteur de l'académie de Rennes, du mois d'août 1854 à février 1861 . 
à 1880, période où le métier est exclusivement masculin ${ }^{4}$. L'étude des dossiers personnels des inspecteurs, conservés aux Archives nationales, et le croisement de ces sources avec les documents des fonds départementaux permettent de cerner l'origine et la nature du corps dans l'académie. Ils font également émerger trois temps forts, dont le premier embrasse les années 1835-1840 du règne de Louis-Philippe, au cours desquelles sont mis en place, dans chaque département, les premiers inspecteurs des écoles ${ }^{5}$. Les années 1860-1865 du Second Empire apparaissent comme le second moment-clé. Ces deux années, 1860 et 1865, permettent en effet le repérage des personnels en exercice. En 1860, le concours des instituteurs donne lieu à la rédaction de mémoires annotés par les inspecteurs primaires, ce qui fournit les noms de ceux qui sont en exercice. En 1865, le recensement ministériel de tous les inspecteurs primaires en activité précise la composition des circonscriptions et le nom de leurs titulaires. Ce créneau chronologique constitue également un moment essentiel de l'histoire de l'inspection primaire en France, au cours duquel le réseau administratif de l'école primaire se consolide pour plus d'un siècle, où les inspecteurs, plus nombreux, exercent depuis 1850 à l'échelon des arrondissements ${ }^{6}$. Enfin, une troisième période, celle des débuts de la Troisième République, est un temps de profond renouvellement du corps d'inspection : $80 \%$ des inspecteurs en place dans l'académie de Rennes, à la fin du Second Empire, quittent leurs fonctions entre 1873 et 1880.

La réflexion sur les différentes missions des inspecteurs et sur les moyens de les réaliser interroge sur la contradiction apparente, chez ces fonctionnaires de l'Ouest, entre des attaches régionales fortes d'une part, l'obligation du service de l'État et des gouvernements au pouvoir, d'autre part. Le statut de fonctionnaire et la prestation de serment aux différents régimes impliquent la mise en œuvre de mesures décidées par le pouvoir central, amenant la question de la portée de leur action administrative et pédagogique, le degré de leur participation aux luttes entre les forces d'opposition traditionnelles et l'État.

\section{Un corps d'inspection primaire, ancré dans l'Académie}

Après la Révolution de 1830 et le vote de la loi Guizot, au sein des académies, se met en place un contrôle ponctuel des écoles primaires, grâce à la grande inspection de l'été 1833, demandée par Guizot, après le vote de sa loi du 28 juin précédent. Deux ans plus tard, l'inspection primaire est établie définitivement, dans un cadre départemental. En Bretagne, comme

4. FERRIER, Jean, Les inspecteurs des écoles primaires, 1835-1995, Paris, L'Harmattan, 1997, tome 1, p. 325 : "La fonction n'est accessible aux femmes qu'à partir de 1882. "

5. 84 nouveaux inspecteurs au cours de l'année 1835-1836.

6. Les documents, concernant l'année 1865 ont déjà été repérés et utilisés par Jean Ferrier pour sa thèse sur l'ensemble du corps des inspecteurs des écoles primaires français, entre 1835 et 1995 : FERRIER, Jean, Les inspecteurs des écoles primaires, 1835-1995, Paris, L'Harmattan, 1997, tome 1, p. 19. 
dans le reste de la France, les départements de l'académie de Rennes disposent chacun, au cours de l'année 1835-1836, d'un inspecteur des écoles auquel s'ajoute, quelques années plus tard, un sous-inspecteur ${ }^{7}$. Ce dernier est parfois nommé très tardivement. En 1839, l'inspecteur des écoles de Loire-Inférieure demande à quitter ses fonctions parce qu'il ne dispose pas encore de sous-inspecteur pour l'assister ${ }^{8}$.

La législation, mise en place en 1850, au moment de la loi Falloux, redéfinit les circonscriptions d'inspection primaire en les multipliant, l'arrondissement devenant l'unité de base et non plus le département ${ }^{9}$. Elle modifie la composition du corps par un développement du recrutement (théoriquement un inspecteur par arrondissement) et une épuration ${ }^{10}$.

\section{La prédominance du recrutement académique}

Si de 1808 à 1850, l'académie bretonne comprend les cinq départements de la Bretagne historique, cela n'est plus vrai, à partir de la loi Falloux. Les limites de l'académie de Rennes se modifient, la faisant éclater en 5 académies départementales en 1850, débordant sur la Mayenne et le Maineet-Loire, avec sept départements à partir de la loi de $1854^{11}$. Pourtant, l'o-

7. Ordonnance du 26 février 1835 établissant dans chaque département un inspecteur spécial de l'instruction primaire. Lettre du ministre Guizot aux inspecteurs, 13 août 1835; ordonnance du 13 novembre 1837 sur la création des sous-inspecteurs (un dans 57 départements, deux dans 4 départements).

8. AN F17/21313 : dossier Jean-Marie MERPAUT. Lettre du sous-inspecteur de l'Oise, Merpaut, au ministre, 15 mai 1850 : « N'ayant pas de sous-inspecteur et pensant que je ne pouvais en obtenir, je quittai, en 1839, les fonctions d'inspecteur primaire que j'exerçais depuis trois ans dans le département de Loire-Inférieure, trouvant qu'un seul fonctionnaire ne pouvait remplir, dans toute leur étendue, les obligations que cette place imposait. " Dix places nouvelles de sous-inspecteurs seront créées à compter du 1-1-1848.

9. L'article 20 de la loi du 15 mars 1850 , mis à exécution le $1^{\text {er }}$ septembre suivant, précise qu'il y a, en principe, autant d'inspecteurs primaires que d'arrondissements. GOEDERTABAJI, Nathalie, Le service public de l'Instruction primaire, sous le Second Empire : l'exemple de l'académie d'Aix, Thèse de droit, Université Panthéon-Assas (Paris 2), 1995, p. 225. C'est la législation Falloux qui fait véritablement des inspecteurs primaires des agents reconnus de l'Instruction publique. Le décret du 29 juillet 1850 leur donne un statut (recrutement, avancement, attributions). Tout chef d'établissement refusant de se soumettre à l'inspection peut être traduit devant le tribunal correctionnel de l'arrondissement et condamné à une amende de $100 \mathrm{~F}$ à $1000 \mathrm{~F}$ (loi du 15 mars 1850, art. 22).

10. Roux, Christian, L'inspection primaire et la surveillance de l'école en France au XIX siècle, Doctorat d'État, Aix-Marseille I, 1994, p. 257-259. Sur les 65 inspecteurs primaires nommés en 1835, 28 (43\%) sont déplacés, suspendus, révoqués ou démissionnent d'euxmêmes (16 pour raisons politiques, 4 pour ne pas avoir établi de bonnes relations avec le clergé, 3 pour incompétence, 3 pour immoralité et 2 sans raison connue). En 1850, et suite au découpage des départements en circonscriptions d'inspection, sur les 65 inspecteurs d'origine, seuls 13 inspecteurs deviennent inspecteurs d'arrondissement, tandis que 26 sont appelés à d'autres postes : 10 directeurs d'écoles normales, 2 inspecteurs d'académie, 5 secrétaires d'académie, 5 principaux de collège. D'autres s'orientent vers d'autres carrières : un notaire, un juge de paix, un délégué à l'administration centrale, un préfet, etc.

11. L'article 7 de la loi sur l'enseignement du 15 mars 1850 établit une académie dans chaque département. Quatre ans plus tard, la loi sur l'Instruction publique du 14 juin 1854 
rigine locale de ces fonctionnaires, bien qu'en recul, continue de dominer jusqu'à la Troisième République. On recrute les inspecteurs des écoles primaires bretonnes, principalement en Bretagne. En 1835-1836, les premiers inspecteurs spéciaux des écoles des cinq départements de l'académie de Rennes sont majoritairement bretons (cinq sur sept) ${ }^{12}$. Les deux venus de l'extérieur ne demeurent respectivement que deux mois et deux ans dans l'académie ${ }^{13}$. Malgré l'instabilité initiale du corps, engendrée par la précipitation de certaines nominations et la précarité de l'exercice des fonctions dans des circonscriptions étendues à l'ensemble d'un département, le corps se stabilise dans la seconde décennie de la monarchie de Juillet.

Un quart de siècle plus tard, pour la période 1860-1865, sur 25 inspecteurs primaires exerçant dans les sept départements de l'académie de Rennes, 64 \% sont nés dans l'un des départements de l'académie. Si on restreint l'étude à la seule Bretagne historique, sur les 16 inspecteurs qui ont la responsabilité des circonscriptions en 1865, 56 \% (9 sur 16) sont natifs de l'un des cinq départements bretons, mais 4 seulement sur 16 (25\%) exercent dans leur département de naissance, prouvant une incontestable mobilité de ces fonctionnaires. Cependant, cette mobilité limitée, intra-académique, est suffisante pour imposer l'idée de " service public ${ }^{14}$ ». Ce terme de "service public " apparu vers 1835 , donc contemporain de la naissance de l'inspection primaire, s'applique non seulement aux institutions administratives mais concerne des missions d'intérêt général, donc des obligations, parmi lesquelles une certaine indépendance et une objectivité de jugement, qui supposent la mobilité et l'éloignement des origines familiale et scolaire.

La particularité linguistique et politique de la Bretagne semble expliquer, au moins partiellement, une mobilité plus réduite et donc un enracinement plus grand des inspecteurs primaires que dans d'autres régions françaises. Lors de l'enquête du ministre sur les inspecteurs, en 1865, dans les trois départements bretonnants (Finistère, Morbihan, Côtes-du-Nord), 2 inspecteurs sur 3 sont originaires de la Basse-Bretagne. Dans les deux autres départements, les inspecteurs étrangers à la Bretagne sont plus nombreux; en Ille-et-Vilaine, deux inspecteurs sur quatre et en Loire-Inférieure, 2 sur 3.

(article 1) divise la France en seize grandes circonscriptions académiques, la composition précise de chaque académie étant fixée par le décret sur l'organisation des académies du 22 août 1854 (article 1).

12. Dans les Côtes-du-Nord, Louis Campion, né dans ce département, dans le Finistère, Louis Calloc'h, originaire de l'île de Groix (Morbihan) sont bretons, de même que, Hellier et Audic, le premier né à Hillion (Côtes-du-Nord), le second à Napoléonville. En mars 1836, Jean-Marie Merpaut, né à Uzel, dans l'arrondissement de Loudéac, succède à l'inspection de Loire-Inférieure, Julien Harlé, démissionnaire après une première tournée ". L'inspecteur du Morbihan fait exception, en venant des Deux-Sèvres.

13. AN F17/20931 : dossier Julien Harlé, inspecteur des écoles de Loire-Inférieure d'octobre à décembre 1835. AN F17/20417, dossier Auguste Chevreau, inspecteur des écoles du Morbihan, de 1835 à 1837.

14. REY, Alain (dir.), Dictionnaire historique de la langue française, Paris, Le Robert, édition petit format, tome 3 , p. 3485 . 
En comparant la carrière de tous les inspecteurs ayant exercé dans les sept départements de l'académie de Rennes, entre 1860 et 1865, on remarque qu'ils ont une expérience antérieure d'enseignant assez longue, un peu plus de 11 ans en moyenne, avec des maxima de 19 à 26 ans. Sur la vingtaine d'inspecteurs affectés dans les départements de la Bretagne historique, une très petite minorité a exercé d'autres professions avant d'entrer dans l'enseignement. On relève trois cas sur 19 : un sous-officier ${ }^{15}$, un auxiliaire de chirurgie ${ }^{16}$ et un avocat reconverti dans l'enseignement pour raisons de santé ${ }^{17}$. Les inspecteurs primaires sont donc, majoritairement, des professionnels de l'enseignement

\section{Stabilité et longévité professionnelle.}

Une autre particularité des inspecteurs primaires se dégage de l'enquête ministérielle de 1865; les natifs de l'un des sept départements de l'académie de Rennes demeurent, en moyenne, 8 années et sept mois par poste occupé (pour les seuls bretons, 8 années et cinq mois). Ceux qui sont nés à l'extérieur de l'académie sont plus mobiles. Au cours de leur carrière, ils restent, en moyenne 5 années dans le même poste. Cependant, ces moyennes masquent une autre particularité des parcours professionnels. Tous les inspecteurs bretons, exerçant dans l'académie de Rennes, à la fin du Second Empire, ont un profil de carrière semblable : ils occupent successivement entre 3 et 4 postes d'inspection, exercent certes dans plusieurs arrondissements, mais très longtemps au sein d'un même département. La durée moyenne de leurs fonctions, dans l'un des cinq départements bretons où ils exercent le plus longtemps, est de 20 années et demie. Joseph Rousselot, natif des Côtes-du Nord, qui accède aux fonctions d'inspection dès 1846 et prend sa retraite en 1881 exerce 32 ans dans son département de naissance et 3 ans dans le département voisin de l'Ille-et-Vilaine. Nicolas Nédélec, né dans le Finistère, occupe des fonctions d'inspection pendant 38 ans, de la monarchie de Juillet à la Troisième République, dont 19 ans dans les Côtes-du-Nord et 19 ans dans son département d'origine. Joseph

15. AN F17/20514 : dossier Pierre DAMY. Né en 1810 à Vieux-Vy (Ille-et-Vilaine), il fait son service militaire de 1831 à 1838 comme engagé volontaire au $6^{\text {e }}$ régiment d'infanterie légère. D'après les registres matricules des Archives de la guerre, il est caporal, puis sergent. Il entre dans l'enseignement en 1838 comme professeur dans une pension libre de Rennes, puis comme instituteur à l'école primaire annexée au Lycée de Nantes (1844-1852).

16. AN F17/21354 : dossier François MonTIER. Né en 1808 à Saint-Brieuc, il est successivement auxiliaire-chirurgien à Brest (1829-1830), maître d'études à Ancenis (mai-octobre 1832), directeur de l'EPS de Saint-Brieuc (1832-1840). Il est nommé inspecteur des écoles des Côtes-du-Nord en 1844 et poursuit sa carrière dans l'inspection. Il quitte le corps en 1869 .

17. AN F17/21105 : dossier Henri Marie Marcel LeBourgeols. Né en 1832 à Avranches (Manche), il fait des études secondaires, obtient le bac ès Lettres et poursuit ses études en droit. Licencié, avocat, il entre dans l'enseignement en 1861 et exerce au Lycée de Nantes. Souffrant d'une maladie du larynx, il souhaite s'orienter vers une carrière administrative. À la mort prématurée de l'inspecteur Poulain, il devient inspecteur de l'arrondissement de Savenay (1864-1868). 
Lequinquis, né dans la partie bretonnante des Côtes-du-Nord, se voit attribuer un poste de sous-inspecteur dès 1838 et ne quitte ses fonctions qu'en 1874. Sur 36 ans de carrière, 26 années se déroulent dans le département du Finistère, 7 dans le Morbihan, 3 en Ille-et-Vilaine. Pierre Hamon, originaire des Côtes-du Nord, est inspecteur de ce département pendant 20 années sur un total de 23 ans de carrière. François Colomb et JeanBaptiste Spal, bretons d'origine, font presque toute leur carrière, respectivement 22 ans et demi et 20 ans, dans le seul département du Maine-etLoire, l'un des sept départements de l'académie de Rennes.

La faible mobilité géographique et la longévité professionnelle se conjuguent pour entraîner le vieillissement du corps, très perceptible sous le Second Empire. Alors que pour la France entière, en 1865, les 40-59 ans représentent, d'après les travaux de Jean Ferrier, les $2 / 3$ du corps ${ }^{18}$, la Bretagne a un corps d'inspection primaire, en moyenne, bien plus âgé, les 40-59 ans regroupant plus de $77 \%$ du corps (11 points de plus). En 1865, les moins de 40 ans sont 4 , tous venus de l'extérieur de l'académie de Rennes. Ce poste en Bretagne est, pour eux, le premier ou le second, donc un poste de début de carrière. Sur ces quatre inspecteurs, un seul, originaire des Deux-Sèvres s'installe durablement en Loire-Inférieure, principalement à Nantes, tremplin pour Paris. Les autres demeurent de 3 à 5 ans seulement en Bretagne, avant d'obtenir un autre poste. Le renouvellement est donc faible. Parmi les inspecteurs et les sous-inspecteurs déjà en poste dans les années 1840 de la monarchie de Juillet, en dépit des décès prématurés, de l'épuration de 1850, des mutations, 50 \% de ces inspecteurs sont encore en fonctions en Bretagne en 1859-1860, 7 inspecteurs sur 16 en 1865 (43\%). C'est également parmi ces mêmes inspecteurs que se recrute, à partir de 1844 et jusqu'en 1877 le directeur de l'école normale régionale ou interdépartementale bretonne. En 33 ans, ils ne sont que deux à occuper ce poste, le premier inspecteur des écoles des Côtes-du-Nord, Louis Campion (directeur de 1844 à 1865), puis le finistérien Victor Bléas (1865-1877). Grâce à une longévité professionnelle exceptionnelle et à une mobilité intra-académique dominante, à une mainmise de ces inspecteurs locaux sur la formation des maîtres laïcs en Bretagne et sur le contrôle des écoles, cette première génération d'inspecteurs modèle l'école primaire en Bretagne, de Guizot à Jules Ferry.

Ce faible renouvellement des membres du corps d'inspection en Bretagne, les attaches régionales fortes de ces inspecteurs exerçant en Bretagne trouvent d'abord leur explication dans la législation en vigueur qui, de la monarchie de Juillet à la III ${ }^{e}$ République, préconise la consultation des autorités académiques avant la nomination ministérielle. La prudence de l'administration centrale joue également un rôle essentiel dans une partie de la France considérée comme " difficile " par tous les régimes en place, à partir de 1830. Les fonctionnaires ayant une longue expérience locale d'enseignant et d'administrateur, bien perçus par les autorités locales et par l'épiscopat sont jugés mieux à même d'agir sur la société et de

18. FERRIER, Jean, Les inspecteurs des écoles primaires, 1835-1995, op. cit., tome 1, p. 330. 
s'entremettre pour l'État auprès des autorités civiles et religieuses, au sein de leur académie d'origine. Sont également préférés des autorités, pour la Basse-Bretagne, les fonctionnaires maîtrisant la langue bretonne, indispensable pour établir des contacts avec les membres des conseils municipaux dans les pays bretonnants ${ }^{19}$. Joseph Calloc'h est un exemple de ces inspecteurs bretonnants, illustrant la spécificité de cette académie. Né dans l'île de Groix en 1795, bachelier et principal du collège de Quimperlé, de 1831 à 1836, il accède à l'inspection des écoles du Finistère sur proposition du recteur de l'académie, parce qu'il dispose d'une solide expérience de 17 ans d'enseignement et d'administration, en tant que précepteur, régent de collège, directeur de pension et principal du collège de Quimperlé (1831-1836). Il a également une connaissance de l'enseignement primaire par ses multiples fonctions de président du jury d'examen des institutrices de l'arrondissement de Quimperlé, de membre du comité supérieur d'arrondissement à partir de 1833. Il a, en outre, été chargé de la grande inspection des écoles primaires de son arrondissement, à l'été de cette même année. Recommandé par le député du Finistère, Tupinier, par celui de LoireInférieure, Bignon, par le maire de Nantes Duval, réputé conciliant et très bien vu des ecclésiastiques, il est le premier inspecteur des écoles du Finistère (1836-1840) ${ }^{20}$, avant d'exercer les mêmes fonctions en LoireInférieure, de 1840 à 1850.

Participe également à ce phénomène de renouvellement limité, la faible attractivité de la Bretagne. On le mesure, dès l'année de création des inspecteurs des écoles, lorsqu'il s'agit de trouver des candidats pour certains départements bretons. En septembre 1835, il ne reste à pourvoir, en France, que la Loire-Inférieure et le Bas-Rhin ${ }^{21}$. Lors de la mise en place des inspections des écoles et la répartition des départements par classes, chaque classe correspondant à un niveau de traitement, sur cinq départements, aucun ne figure en première classe, deux sont placés en deuxième classe, trois en troisième classe ${ }^{22}$. Cette répartition provoque d'ailleurs les

19. AN F17/20054 : dossier Louis AuDIC. Extrait du registre des délibérations du Conseil royal de l'Instruction publique, séance du 4 novembre 1836. Les inspecteurs des écoles primaires, conformément à l'art. 3 de l'Ordonnance royale du 26 février 1835, sont nommés par le ministre de l'Instruction publique, le conseil royal entendu. Leur nom est proposé par le recteur de l'académie, de concert avec le préfet du département qui accueille le nouvel inspecteur.

20. AN F17/20319 : dossier de Joseph Marie CALLOC'H. Lettre du recteur de l'académie de Rennes au ministre, 26 novembre 1834; Lettre du préfet de Loire-Inférieure au ministre, 27 août 1835; lettre du député Bignon au ministre, 26 juin 1836.

21. AN F17/20931 : dossier de l'inspecteur HARLE. Lettre de Harlé, ancien chef d'Institution au ministre de l'Instruction publique, 25 septembre 1835 : "Vous me promîtes une place d'Inspecteur d'Écoles primaires dans un des trois départements restants à pourvoir, l'Oise, la Loire-Inférieure et le Bas-Rhin [...]. Sachant que vous venez de disposer du département de l'Oise, j'ose solliciter de votre bienveillance, la place de la Loire-Inférieure. Je saisis ici l'occasion de vous répéter que mes sentiments religieux sont tels qu'on doit les exiger dans un département où la Religion est observée avec tant de zèle [...]. "

22. Arch. dép. d'Ille-et-Vilaine, 11T 26 : Bulletin Universitaire, $\mathrm{n}^{\circ} 101$ du 3 août 1835, Arrêté $n^{\circ} 934$ du ministre de l'Instruction publique qui divise les inspecteurs de l'Instruction 
protestations des autorités académiques, préfectorales et celles des parlementaires ${ }^{23}$.

\section{Le sous-encadrement et l'obstacle de la distance}

Au moment de la restructuration de l'inspection primaire, en 1850, le nombre des arrondissements en France s'élève à 361 et le budget voté par l'Assemblée législative ne permet de nommer que 300 inspecteurs ${ }^{24}$. Le taux moyen d'encadrement français est de 83,1 \%. L'Ouest se trouve en net retrait. L'article 20 de la loi Falloux, donnant la faculté de réunir plusieurs arrondissements sur l'avis du conseil académique, quinze ans plus tard, la Bretagne ne disposant toujours que de 16 inspecteurs pour 25 arrondissements, elle conserve un faible taux d'encadrement territorial $(64 \%)^{25}$. Beaucoup de circonscriptions de l'académie de Rennes, surtout en Bretagne, comprenant deux arrondissements et parfois quelques cantons d'un troisième, sont immenses et difficiles à parcourir. À la fin du Second Empire, la circonscription de Napoléonville-Ploërmel, privée du chemin de fer, a une longueur d'ouest en est de 140 kilomètres, ce qui rend le service de l'inspecteur exténuant. L'inspecteur de la circonscription de Saint-Brieuc estime, en 1865 qu'il parcourt 2400 à 2500 kilomètres pendant les 150 journées d'inspection annuelle et que cela représente 16 kilomètres par jour. Dans le Finistère, à la mauvaise qualité des chemins, s'ajoute l'impossibilité de trouver un gîte ailleurs que dans les chefs-lieux de cantons. L'inspecteur de Morlaix n'a aucun moyen de communication à sa disposition entre sa résidence administrative et les trois cantons de l'arrondissement de Châteaulin dont il a la charge, et qui sont éloignés de 30, 48 et 72 kilomètres de sa résidence. L'inspecteur de Vitré-Fougères peut utiliser les 30 kilomètres de voies ferrées traversant une partie de l'arrondissement de Vitré, le service de voiture entre les deux chefs-lieux d'arrondissements, mais pour l'essentiel de ses inspections il n'a que la marche à pied comme moyen d'accéder aux

primaire en 3 classes et qui fixe le traitement affecté à chaque classe et la somme allouée pour frais de tournée. Les inspecteurs de première classe sont placés dans 9 départements dont aucun de l'académie de Rennes. Les inspecteurs de deuxième classe sont placés dans 15 départements dont l'Ille-et-Vilaine, la Loire-Inférieure. Les inspecteurs de troisième classe sont placés dans 62 départements dont les Côtes-du-Nord, le Finistère, le Morbihan, la Mayenne. Les traitements des inspecteurs sont ainsi réglés : $1^{\text {re }}$ classe : $2000 \mathrm{~F} ; 2^{\mathrm{e}}$ classe : $1800 \mathrm{~F} ; 3^{\mathrm{e}}$ classe : $1500 \mathrm{~F}$ (1600 F par arrêté du 29 décembre 1837).

23. AN F17/9612 : Lettres du recteur de l'académie de Rennes au ministre, 10 janvier 1840, 17 mars 1842, 8 avril 1843; lettres du préfet des Côtes-du-Nord au ministre, 20 mars 1843, mars 1842; Séance du Comité supérieur d'Instruction primaire du 8 mars 1843.

24. Arch. dép. d'Ille-et-Vilaine, 10T 4 : Registre du conseil académique de Rennes, séance du 4 octobre 1850.

25. AN F17/9294 : État nominatif des inspecteurs de l'instruction primaire, 1865. Ces documents, demandés par le ministre fournissent, pour chaque département de l'académie, le nom des circonscriptions, la description de chaque arrondissement (population, superficie, nombre d'écoles et de salles d'asile, les difficultés de parcours et d'inspection), l'état civil et les états de service de chaque inspecteur. 
écoles. L'inspecteur de l'arrondissement de Nantes et Paimbœuf qui réside au chef-lieu départemental a plus de chance, mais doit changer fréquemment de moyen de locomotion : chemin de fer dans le canton de Carquefou, bateau à vapeur sur la Loire et sur l'Erdre, enfin les voitures publiques ${ }^{26}$.

La situation excentrée de la Bretagne par rapport au reste de la France, les difficultés de l'exercice de l'inspection en Bretagne ont d'autres conséquences, parmi lesquelles la difficile acculturation des inspecteurs venus de l'extérieur. Julien Harlé, venu de Seine-et-Oise, donne sa démission après sa première tournée en Loire-Inférieure, à l'automne $1835^{27}$. Eugène Barthélémy, originaire du Cher, inspecteur à Lorient après avoir commencé sa carrière dans les arrondissements de Corte et Calvi, se dit autant étranger en Bretagne qu'en Corse. Il vit replié dans un petit village du Morbihan ${ }^{28}$. Frédéric Decourt, ancien instituteur de la Nièvre, nommé à Morlaix en 1855 voit sa circonscription s'élargir à plusieurs cantons de l'arrondissement de Châteaulin à partir de 1859. Il multiplie les courriers pour fuir un arrondissement " rude, humide, accidenté ", " les fatigues inouïes " de ses tournées ${ }^{29}$ et les relations difficiles avec le maire de Morlaix ${ }^{30}$. Cet inspecteur est remplacé par le finistérien, Nédélec en 1863. Cependant, ces inspecteurs, étrangers à la Bretagne, même quand leur séjour est bref, offrent quelques exemples de brillante réussite. Le bourguignon François Serbource, pendant ses trois ans à Brest, laisse un excellent souvenir aux édiles locaux et inspire un grand respect chez les instituteurs de cette partie du Finistère ${ }^{31}$.

26. AN F17/9294 : État nominatif des inspecteurs de l'instruction primaire, académie de Rennes, 1865.

27. AN F17/20931 : Lettre de l'inspecteur des écoles de Loire-Inférieure, Harlé, au recteur de l'académie de Rennes, Nantes, le 12 décembre 1835 : « J'ai voyagé pendant dix jours et pendant tout ce temps, je n'ai rencontré que des chemins affreux dans lesquels j'ai failli vingt fois perdre mon cheval et rester moi-même au milieu de l'eau et de la boue. Logé dans de mauvais cabarets où je ne trouvais pour coucher que de la paille, et pour toute nourriture que du mauvais pain noir et du fromage, je n'ai pu résister à une telle manière de vivre, et après avoir terminé ma visite à la Mouzinière, j'ai résolu d'abandonner la partie à plus fort et plus courageux que moi. Une telle corvée, que je déclare impossible dans cette saison, est au-dessus de mes forces et je vous prie d'accepter ma démission. "

28. AN F17/20098 : rapport de l'inspecteur général Rapet, juin 1866 : « Peut-être va-t-il un peu loin, et surtout veut-il aller trop vite, ce qui paraît insolite dans un pays où on n'a pas l'habitude de se presser."

29. Arch. dép. du Finistère, 1T 399 : lettre de l'inspecteur Decourt à l'inspecteur d'académie, 5 octobre 1863 .

30. Arch. dép. du Finistère, 1T 399 : lettre de l'inspecteur Decourt à l'inspecteur d'académie, 16 août 1859 .

31. AN F17/21720 : dossier François Erasme Serbource. Né en 1831 à Poinçon (Côted'Or), ancien élève de l'école normale de Dijon et instituteur de 1854 à 1860, devient inspecteur en 1860. Après deux ans en Lozère et deux ans en Ardèche, il est envoyé en Bretagne où il a la charge de l'arrondissement de Brest (1864-1867). Si d'après le recteur de l'académie de Rennes, il ménage trop peu les autorités locales, dans une lettre au ministre, du 18 décembre 1866, l'adjoint au maire de Brest écrit : " C'est à ses inspirations, à ses conseils que sont dues les améliorations sérieuses récemment introduites dans les écoles communales de Brest [...] Le respect et l'affection des instituteurs dont il s'est entouré, la confiance de l'Administration municipale qu'il a su conquérir, ont produit des résultats. M. Serbource est président de la Société de Secours mutuels des Instituteurs 


\section{Une carrière plus ouverte aux instituteurs normaliens et aux bretonnants}

La forte attache du corps de l'inspection primaire au milieu régional d'origine (52,6\% des inspecteurs en fonctions dans les cinq départements de la Bretagne historique en 1865 sont nés en Bretagne) est complétée par une autre particularité à double facette. La première est non spécifique à l'académie de Rennes, c'est l'ouverture de l'inspection aux instituteurs ${ }^{32}$. En Bretagne, comme ailleurs, les inspecteurs, à l'origine, très majoritairement issus de l'enseignement secondaire, sont de plus en plus recrutés parmi les instituteurs chevronnés, au point de devenir majoritaires après 1850. Cette évolution, encouragée dès la monarchie de Juillet, est liée à une meilleure formation des instituteurs qui peuvent, pour une élite, accéder au brevet supérieur, tremplin prévu par les textes officiels de 1845 et 1846 pour une direction d'école primaire supérieure, un poste d'inspecteur des écoles ou de directeur d'école normale. En 1865, si 36,8 \% des inspecteurs de l'académie de Rennes ont une formation secondaire et ont effectué un début de carrière au sein de l'enseignement secondaire, principalement en collège, plus de $63 \%$ ont exercé la profession d'instituteur avant d'accéder à l'inspection. En considérant les dossiers personnels de 25 inspecteurs en fonctions dans l'académie de Rennes, entre 1860 et 1865, 16 d'entre eux (64\% = environ deux tiers) ont fait une scolarité en école normale. La répartition des niveaux de diplôme entre les inspecteurs de Bretagne est proche de la moyenne française. En 1865, il y a $40 \%$ de bacheliers parmi les inspecteurs en exercice sur l'ensemble du territoire français, 38 \% en Bretagne, $6 \%$ de licenciés en France, 4,5\% en Bretagne. Les titulaires du seul brevet supérieur sont environ 53 \% pour l'ensemble de la France, 55 \% pour les inspecteurs des cinq départements de la Bretagne historique.

Cependant, la Bretagne se distingue des autres parties de la France par le fait qu'un tiers de ses inspecteurs en fonctions sous le Second Empire, qu'ils soient Finistériens, Morbihannais, de Loire-Inférieure, des Côtes-duNord ou d'Ille-et-Vilaine, sort de l'école normale régionale de Rennes, alors que dans le reste de l'hexagone, le système des écoles normales départe-

et Institutrices du Finistère : ces fonctions lui ont été dévolues à la presque unanimité des suffrages des sociétaires [...]."

32. L'ouverture du corps d'inspection des écoles aux enseignants du primaire constitue, dès l'origine, la seule possibilité de promotion pour une élite d'instituteurs, le brevet supérieur et l'examen d'aptitude aux fonctions d'inspecteur et de directeur d'école normale étant les conditions nécessaires depuis les textes de 1845-1846, mais non suffisantes, pour avoir accès à la candidature. L'appréciation des supérieurs hiérarchiques et les recommandations politiques font toujours la décision. Le passage des fonctions d'instituteur à celles d'inspecteur demande des diplômes (Brevet supérieur ou brevet complet, ainsi que la réussite à un examen spécifique), des recommandations, mais également et surtout beaucoup de temps car les postes sont peu nombreux, bien moins nombreux que ne le prévoit la législation de 1850. La demande est beaucoup plus forte que l'offre. La III République exclut momentanément les instituteurs de l'accès à l'inspection par le décret du 23 décembre 1882, avant de leur offrir des dérogations à partir de 1887. Parmi les inspecteurs, une majorité sort d'une école normale. 
mentales domine depuis 1838. Le réseau de relations professionnelles - et amicales - est géographiquement beaucoup plus large en Bretagne qu'ailleurs. Au sein même de la grande académie de Rennes, après 1854, le contraste est fort entre les cinq départements bretons d'une part et la Mayenne et le Maine-et-Loire de l'autre, où le réseau de relations professionnelles est restreint au département ${ }^{33}$. La situation bretonne est donc unique et l'existence d'un réseau de relations interdépartementales entre les membres du corps d'inspection breton, réparti entre les arrondissements et la direction de l'école normale interdépartementale joue un rôle essentiel à certains moments difficiles, en particulier en 1850, lorsque la menace pèse sur la survie de l'école normale de Rennes ${ }^{34}$.

En Bretagne, l'inspection primaire présente un autre aspect singulier, celui d'un accès au corps plus facile pour les bretonnants. Sur 17 inspecteurs nés en Bretagne et ayant exercé dans l'Ouest sous le Second Empire, 14 sont nés dans le Finistère ou dans les Côtes-du-Nord, dans ce dernier département à l'ouest de la ligne Saint-Brieuc-Vannes. Le seul inspecteur morbihannais, Lorans, mort dans l'exercice de ses fonctions, est originaire de la partie bretonnante et est remarqué par la hiérarchie académique pour sa connaissance de la langue bretonne ${ }^{35}$. Au sein de l'échantillon, on ne repère que deux inspecteurs de l'est breton, un d'Ille-et-Vilaine et un de Loire-Inférieure. Si on ne tient pas compte des inspecteurs issus de l'enseignement secondaire, en se limitant aux inspecteurs issus de la première génération de normaliens bretons, 11 sur 13 sont originaires des pays bretonnants ${ }^{36}$. L'inspection primaire constitue donc, en Bretagne, une véritable perspective de carrière pour une élite d'instituteurs bretonnants, ayant la double particularité d'avoir été formés en Haute-Bretagne, à l'école interdépartementale de Rennes et de maîtriser la langue bretonne. Sous le Second Empire, la langue bretonne étant présentée, par les inspecteurs

33. Ces deux départements ont leur école normale départementale depuis la monarchie de Juillet.

34. Ceci se remarque particulièrement dans les échanges de correspondance entre ces fonctionnaires bretons. On peut citer, entre autres, Arch. dép. du Finistère 1T817 : rapport confidentiel du directeur de l'école normale primaire, Louis Campion, à l'inspecteur des écoles du Finistère Lequinquis, pour la défense de l'école normale de Rennes, 13 juin 1850. Le directeur de l'école normale signe "votre ami et compatriote dévoué ". Louis Campion et Joseph Lequinquis sont nés sous le Premier Empire, dans le même département, les Côtes-du-Nord. Ils appartiennent à la première génération du corps d'inspection des écoles en Bretagne; Lequinquis a occupé ces fonctions dans le Morbihan et le Finistère, Campion dans les Côtes-du-Nord. Louis Campion est directeur de l'école normale de Rennes (1844-1865) et L'inspecteur Lequinquis est un ancien élève de la première promotion de cette même école (1831-1832).

35. AN F17/21197 : dossier Jean-Jacques LoRANS, rapport de l'inspecteur général Villemereux, Vannes, le 26 septembre 1859 : «Un des meilleurs élèves de l'excellente école normale de Rennes [...]. Il est enfant du pays, il parle bas-breton et ses divers dialectes avec une facilité rare, même chez les indigènes, qui lui assure la faveur populaire et de l'autorité civile et ecclésiastique dans les campagnes. "

36. NiCOLAS, Gilbert, L'école normale primaire de Rennes et la première génération de normaliens en Bretagne (1831-1852), thèse de doctorat, Paris 4, 1992, vol. 2, p. 542 et 543. 
eux-mêmes, dans leur notice individuelle comme " une langue étrangère vivante ${ }^{37}$ ".

Au cours du XIX ${ }^{\mathrm{e}}$ siècle, le corps des recteurs d'académie, se laïcise à partir de 1830, s'ouvre aux non bretons à partir de $1847^{38}$, évolution suivie par les inspecteurs d'académie. Le corps des inspecteurs primaires est donc le dernier échelon administratif de l'enseignement primaire à conserver ce lien fort avec le milieu d'origine géographique de ses membres.

\section{Réseaux, action et médiations}

Les missions de l'inspecteur primaire, fonctionnaire subalterne, seul membre des corps d'inspection en contact régulier avec les instituteurs et le terrain communal, se trouvent au cœur des luttes scolaires, politiques et linguistiques de l'Ouest. De la monarchie de Juillet à la III ${ }^{\mathrm{e}}$ République, l'école est l'un des terrains d'affrontement régulier entre le clergé, l'épiscopat et les congrégations d'un côté, l'administration universitaire et les instituteurs laïques de l'autre ou bien les libéraux ou les républicains d'un côté, les conservateurs et ce que les républicains appellent dans la décennie 1870-1880 "le parti clérico-légitimiste ", de l'autre. S'il est possible de distinguer une alternance de phases de grandes tensions (1830-1839, 18481852, 1859-1863) et des périodes plus calmes, l'importance de l'enjeu constitué par le contrôle de l'école donne à cette lutte en Bretagne, un caractère permanent, des années 1830 aux années 1880. Cet affrontement se lit dans les rapports de l'inspection générale, dans les rapports des recteurs, des inspecteurs d'académie, dans la correspondance et les mémoires des instituteurs publics de 1861.

Sous l'Empire autoritaire, l'inspecteur général ecclésiastique, Vincent, en tournée en 1855 et 1857, évoque " le clergé breton peu bienveillant pour l'Université ${ }^{39}$ ", découvre " une guerre sourde mais incessante " que se livrent l'administration de l'État et l'autorité épiscopale. Évoquant l'enseignement primaire, il rappelle que cette partie de la France " est peu disposée à l'observation des règlements universitaires ${ }^{40}$ ". La terminologie employée relève du vocabulaire de guerre. En juin 1857, lors de la visite de la circonscription de Lannion, l'inspecteur général écrit : " Depuis deux ans aucune école congréganiste ne s'est élevée sur les ruines d'une école laïque ${ }^{41}$. " Le rapport concernant la circonscription de Loudéac traite des congrégations qui "n’ont ni gagné ni perdu du terrain ".

37. AN F17/20054 : dossier Louis AuDIC, notice individuelle rédigée par l'inspecteur primaire, 1864-1865.

38. À la suite de la mise en congé du recteur breton de l'académie de Rennes, Louis Dufilhol, ce dernier est remplacé, en 1847, par l'inspecteur général pour l'enseignement du droit, Louis Firmin Laferrière.

39. AN F17/21389 : dossier NEDELEC, rapport du $1^{\text {er juin } 1857 .}$

40. AN F17/20918 : dossier personnel de l'inspecteur Louis GuYOMARD. Rapport de l'inspecteur général Vincent sur l'inspecteur primaire de Fougères, juin 1857.

41. AN F17/21389 : dossier NEDELEC, rapport du $1^{\text {er juin } 1857 .}$ 
À l'échelon de l'administration académique, les recteurs d'académie de la monarchie de Juillet, d'origine bretonne de 1830 à 1847, livrent un combat difficile aux congrégations enseignantes, tout particulièrement à celle de l'Instruction chrétienne de Jean-Marie de Lamennais. Avec le Second Empire, la lutte ne se dément pas. Au début de l'année 1859, dans son " rapport politique trimestriel ", le recteur de l'académie de Rennes, Mourier insiste sur la question religieuse, question centrale, question vitale, autant pour le régime que pour le développement de l'école primaire, en particulier dans l'Ouest.

"Les congrégations, liées souvent au parti légitimiste, travaillent les communes pour évincer les instituteurs ou institutrices laïques. Il y a là un véritable danger, non pour l'instruction primaire en elle-même, mais pour le gouvernement impérial qui n'aurait plus de points d'appui dans les communes rurales le jour où les instituteurs laïques disparaîtraient ${ }^{42}$."

Le recteur emploie cette même terminologie de combat, résurgence de l'époque révolutionnaire. Lors de la préparation du voyage de Napoléon III en Bretagne, il oppose les " Chouans du Morbihan ", à " la population libérale de Saint-Brieuc ${ }^{43}$ ".

Au-delà de la terminologie, les actes d'hostilité et les affaires sont nombreux qui traduisent des moments de grandes tensions. Après les affaires des années 1830 (l'affaire Barbot, l'affaire Touron à Janzé), le mécontentement de l'épiscopat à partir du vote de la loi de 1854 est perceptible dans les comptes rendus des séances du Conseil académique. L'affaire romaine accentue les luttes entre le clergé et les représentants de l'Instruction publique, luttes qui entraînent un fort absentéisme de l'épiscopat au sein des instances académiques puis la démission de Monseigneur Brossays-Saint-Marc du Conseil académique en 1860. Lors des élections, les tensions politiques touchent également le personnel de l'enseignement primaire. Dans une protestation de 1860, adressée au Corps législatif, le candidat légitimiste proteste contre les actes illégaux s'étant déroulés lors de l'élection législative partielle de Dalmas en Ille-et-Vilaine, l'année précédente et ayant impliqué des membres de l'enseignement primaire $^{44}$. L'affaire de l'école du Sel-de-Bretagne ${ }^{45}$ en 1861-1862 et celle de

42. AN F17/2649, microfilm : rapport trimestriel politique du recteur de l'académie de Rennes au ministre, 7 janvier 1859, p. 3 à 5 .

43. AN F17/2649, microfilm : rapport trimestriel politique du recteur de l'académie de Rennes au ministre, 9 juillet 1858, p. 4. Dans le même rapport (page 10), le recteur de l'académie de Rennes représente l'arrondissement de Segré comme une "Petite Vendée ".

44. Arch. dép. d'Ille-et-Vilaine, 1F 1105, Fonds de La Borderie : Protestation de Le Beschu de Champsavin, conseiller à la Cour impériale de Rennes, Paris, Jouaust, 1860, p. 23, 32, 33.

45. Arch. dép. d'Ille-et-Vilaine, 1F 1105, Fonds de La Borderie : Pétition au Sénat de l'Archevêque de Rennes, Vatar, Rennes, 4 février 1862, p. 3 à 5 . Le 31 mars 1861, l'instituteur de la commune du Sel (arr. de Redon), Péan, décède. Le 18 avril suivant, le conseil municipal " à la majorité de huit voix contre deux ", déclare vouloir un Frère, décision confirmée le 25 juillet 1861 (7 voix contre 3). Le $1^{\mathrm{er}}$ novembre 1861, un arrêté du préfet d'Ille-et-Vilaine, Féart, nomme un laïc Oresve, instituteur public de la commune du Sel. L'archevêque de Rennes proteste auprès du ministre Rouland, qui justifie l'action du 
l'école d'Erbrée, en $1864^{46}$ provoquent l'affrontement entre $\mathrm{M}^{\mathrm{gr}}$ Brossays Saint-Marc et le préfet Féart. À la fin du Second Empire, le Conseil général emmené par Arthur de la Borderie, président de la Société pour l'Enseignement libre ${ }^{47}$, refuse une subvention pour l'entretien d'un commis à l'inspection académique, l'inspecteur d'académie de Chateauneuf, ayant vigoureusement défendu l'enseignement laï ${ }^{48}$. Comment les inspecteurs primaires parviennent-ils à remplir leurs différentes missions dans un tel contexte?

\section{Les moyens de l'action}

Bien qu'étant des représentants subalternes de l'État, les inspecteurs disposent de moyens d'action importants. Ils ont d'abord un statut professionnel. Une expérience obligatoire dans l'enseignement et l'obtention d'un examen spécifique permettent l'accès au corps. Depuis 1850, chaque circonscription, théoriquement limitée à un arrondissement, est, géographiquement, plus réduite, donc mieux connue, grâce également à l'obligation de résidence. Le ressort de l'inspecteur primaire est le premier maillon du réseau administratif de l'enseignement primaire, devenu plus dense et plus efficace. Dans la seconde moitié du XIX ${ }^{e}$ siècle, la hiérarchie dans laquelle s'intègre l'inspecteur primaire est mieux définie. Il ne doit rendre des comptes qu'à l'inspecteur d'académie de son département ${ }^{49}$. Le ministre rappelle à l'ordre des préfets qui demandent des rapports aux inspecteurs primaires. L'inspection dispose de traitements hiérarchisés, variant

préfet. L'archevêque Brossays Saint-Marc se tourne alors vers le Sénat auquel il adresse une pétition de 19 pages.

46. Arch. dép. d'Ille-et-Vilaine, 1F 1105, Fonds de La Borderie : Extrait du registre des délibérations du conseil municipal d'Erbrée, 14 novembre 1864 et copie de l'arrêté préfectoral du 17 juin 1864. Le préfet d'Ille-et-Vilaine, Féart, s'oppose, en invoquant le double rapport de la morale et de la salubrité à l'ouverture d'une école libre à Erbrée.

47. Arch. dép. d'Ille-et-Vilaine, 1F 1105 Fonds de La Borderie : Brochure de la Société de l'enseignement libre du département d'Ille-et-Vilaine. Concours des écoles libres, Rennes, Catel, 1869, 24 p. La Société est autorisée le 4 août 1868. Elle a pour but de soutenir le développement de l'enseignement libre (art. I des statuts). Elle est administrée par un conseil ou comité de 20 membres (entre autres Le Beschu de Champsavin, conseiller honoraire à la Cour Impériale, de La Borderie, membre du Conseil général d'Ille-et-Vilaine (Vitré et Rennes), du Marquis de la Bourdonnaye, ancien membre du Conseil Général du Morbihan etc. En dehors des cotisations réglementaires, la Société reçoit tous dons en argent, livres, fournitures scolaires etc. (art. VIII). Dès juillet 1869, la Société compte déjà 700 membres, parmi lesquels l'archevêque Brossays Saint-Marc, l'ancien évêque de Vincennes, $\mathrm{M}^{\mathrm{gr}}$ de La Hailandière, deux députés, 20 conseillers généraux, plusieurs conseillers d'arrondissement, des maires, des magistrats, nombre de propriétaires, de prêtres, d'avocats, de médecins, de professeurs, etc. En 1868-1869, la Société donne des subventions à 25 écoles libres. Elle propage la pétition réclamant la liberté de l'enseignement supérieur. Elle rassemble les matériaux " d'une curieuse histoire de la liberté d'enseignement dans le département d'Ille-et-Vilaine ".

48. Instruction du 31 octobre 1854, relative aux nouvelles attributions conférées aux préfets par la loi du 14 juin 1854, en ce qui concerne l'enseignement primaire.

49. Article 24 du décret sur l'organisation des académies, 22 août 1854. 
entre deux fois et quatre fois le revenu minimum d'un instituteur rural (de $1200 \mathrm{~F}$ en $5^{\mathrm{e}}$ classe à $2400 \mathrm{~F}$ en $1^{\mathrm{re}}$ classe) auxquels s'ajoutent une indemnité départementale et des frais de tournées. Les prescriptions ministérielles imposent une distanciation vis-à-vis des autorités locales et des subordonnés (droit de commensalité limité avec les curés bien sûr, mais également avec les instituteurs). Une direction et une surveillance de l'action de l'inspecteur primaire sont menées par l'inspecteur d'académie de son département. Il est évalué et fait l'objet, chaque année, d'un rapport du recteur de l'académie et, à intervalles plus ou moins réguliers, d'un rapport de l'inspecteur général en tournée ${ }^{50}$.

Dans la seconde décennie du Second Empire, la surveillance se renforce; l'inspecteur général accompagne, parfois pendant plusieurs jours, l'inspecteur primaire dans ses tournées et peut venir former tous les inspecteurs d'un département au chef-lieu, sur des thèmes tels que le mariage de l'instituteur ou la statistique, ce dernier exercice constituant pour les instituteurs et les inspecteurs primaires du Second Empire, une partie de plus en plus importante de leur temps professionnel et, d'après leurs plaintes, un véritable fardeau ${ }^{51}$.

\section{La diversité des missions}

Le corps d'inspection joue un rôle essentiel dans le recrutement des candidats aux fonctions d'instituteur et dans la formation des maîtres. Les directeurs d'écoles normales sont, en effet, à partir de la monarchie de Juillet et progressivement, tous inspecteurs primaires, l'examen de l'inspection ouvrant sur les deux fonctions. Les inspecteurs primaires interviennent également dans la délivrance des diplômes (participation aux commissions d'examen de l'instruction primaire, en particulier pour les différents brevets) dans la formation " continue " au cours de la carrière (conférences pédagogiques initiées sous la monarchie de Juillet et relancées sous le

50. La lecture des dossiers personnels des inspecteurs en fonctions en Bretagne, sous le Second Empire, permet de relever, entre autres, les rapports des inspecteurs généraux Vincent en 1857 et 1858, Villemereux, en 1858, 1859, 1861 et 1863, Rapet en 1864 et 1866, Beaudouin en 1870 et 1871, Beuvain d'Altenheim, en 1872.

51. Ferard, C.-D., Mémoires d'un vieux maître d'école, Delagrave, Paris, 1894, p. 267. AN F17/20730 : dossier de Clément FERARD. Né le 24 mars 1828 à Saint-Aubin-d'Arquenay (Calvados), élève de l'école normale de Caen (sorti en 1846) il est d'abord instituteur public à Formigny (1846-1847), à Saint-Aubin-sur-Mer (1847-1856), puis maître-adjoint à l'école normale de St-Lô (1856-1859) et à l'école normale de Caen (1859-1863). Il entre dans le corps de l'inspection primaire en mars 1863 et est affecté à Brives (Corrèze) en mars 1863, puis immédiatement à Saint-Malo (1863-1868). Il demande très rapidement son retour vers sa région d'origine car il y a laissé sa famille. Il continue sa carrière dans les arrondissements de Pont-Lévêque (1868-1874), Coutances (1874-1877), Pont-Audemer (1877-1878) et termine à Coutances (1878-1887). C'est lors de son séjour dans la circonscription de Saint-Malo, en Ille-et-Vilaine, qu'il participe à une très longue journée consacrée à la statistique, présidée par l'inspecteur général Jean-Jacques Rapet. C'est cet épisode marquant qui est relaté, avec ironie et sans bienveillance pour la haute administration, dans ce passage de ses mémoires. 
Second Empire). Ils assurent le contrôle de l'enseignement primaire public et libre. Par une double tâche, le travail de bureau et les visites sur le terrain, l'inspecteur primaire du $\mathrm{XIX}^{\mathrm{e}}$ siècle établit le lien entre l'abstraction de l'État et la réalité d'une école urbaine ou rurale, entre les instituteurs et le ministère. Il assure la correspondance, frappante de rapidité sous le Second Empire. Il élabore les statistiques. Il participe également à la procédure de construction et de réparation des maisons d'école. Il est, au total, un agent de plus en plus efficace de l'État et de l'uniformisation de l'école.

Le travail de fonctionnaire de l'État combine en Bretagne, plus qu'ailleurs, la défense de l'école publique et des instituteurs laïques. Ceci est vrai sous la monarchie de Juillet (enquête rectorale de 1840 sur le recensement des frères congréganistes et sur leur possession d'un brevet), sous la Seconde République, sous le Second Empire à partir de 1854, plus encore à partir de 1859-1860. Le contexte particulier de l'Ouest, les missions et les moyens mis à leur disposition, la longévité professionnelle des inspecteurs en font, plus qu'ailleurs, des médiateurs.

Leur médiation est d'abord culturelle. Les inspecteurs étant, soit d'anciens instituteurs, soit d'anciens personnels des collèges, ils appartiennent à l'un des rares corps de fonctionnaires qui soit un point de contact entre la culture secondaire et la culture de l'enseignement primaire. Ils témoignent également de la symbiose entre culture laïque et pratiques religieuses. En outre, la plupart des inspecteurs des départements bretonnants parlent les idiomes locaux tout en défendant avec conviction la langue française. L'atout de la pratique linguistique régionale chez les inspecteurs est souligné par la haute administration en même temps que celle-ci se flatte de l'action de certains inspecteurs bretonnants, participant au recul de la langue et des écoles bretonnes au profit du français. L'action du finistérien et bretonnant Victor Bléas parvient à réduire le nombre d'écoles en langue bretonne et reçoit les compliments de l'inspection générale. Son collègue, Joseph Lequinquis, suggère à l'administration académique les moyens de faire progresser la pratique du français en scolarisant les filles, futures mères qui transmettront la langue apprise à leurs enfants, et en sanctionnant les élèves qui parlent breton à l'école. Ces deux personnages constituent des exemples d'inspecteurs bretons de BasseBretagne et bretonnants qui participent activement à la francisation de l'école en Bretagne ${ }^{52}$.

Par leurs connaissances agricoles, historiques, géographiques acquises à l'école normale, plusieurs inspecteurs publient des ouvrages sur la géographie de leur département ${ }^{53}$, ouvrages qui se retrouvent dans les bibliothèques et les écoles de leur département. Certains parviennent à devenir correspondants de sociétés savantes. En Loire-Inférieure, Spal,

52. Arch. dép. du Finistère 1T 68 : lettres de l'inspecteur primaire Bléas à l'inspecteur d'académie du Finistère, 16 septembre 1857 et 3 novembre 1863.

53. Entre autres, J. Rousselot, éd. ViVIER, J. GAultier du MotTAY, Géographie départementale des Côtes-du-Nord, Saint-Brieuc, Guyon, Paris, Hachette, 1862, 844 p. 
récompensé de nombreuses fois pour ses activités en matière de formation agricole est correspondant de la Société académique de Nantes ${ }^{54}$.

\section{La figure de l'inspecteur}

Les rapports des recteurs et des inspecteurs généraux esquissent un portrait ambivalent de l'inspecteur. Supérieur hiérarchique de l'instituteur, il est pourtant rarement lettré, aisé, distingué ou intégré au monde urbain. Il est plus souvent marqué par la simplicité apparente, les manières rustiques, un mariage avec une femme de modeste extraction, une situation pécuniaire précaire. À la jonction de deux cultures, l'inspecteur en Bretagne est tout de même plus proche de la classe la plus nombreuse que de l'élite. Les portraits d'inspecteurs bretons, esquissés par les inspecteurs généraux, dans les rapports rédigés à l'issue de leurs tournées confirment cette proximité du peuple et du monde des instituteurs dont ils sont les supérieurs directs. De la monarchie de Juillet au Second Empire, les inspecteurs généraux évoquent, avec leur plume acérée, "l'enveloppe épaisse " de l'inspecteur de Dinan, Hamon ${ }^{55}$, "les formes peu élégantes " de l'inspecteur du Morbihan Lequinquis " simple et presque rustique pour vivre et réussir au milieu des populations rudes et incultes du Morbihan ${ }^{56}$ ", "la tenue, les manières sans distinction et l'esprit peu élevé " de Damy ${ }^{57}$ en LoireInférieure, "l'écorce un peu rude " de l'inspecteur Spal, originaire de ce même département ${ }^{58}$, "l'esprit un peu lourd " de l'inspecteur Délivré ${ }^{59}$, en charge de l'arrondissement de Vannes. Si les descriptions touchant à l'aspect extérieur des personnages sont rarement flatteuses, la hiérarchie leur reconnaît des qualités spécifiques : une capacité d'adaptation au pays, aux populations, aux autorités. Grâce à leur connaissance du breton pour une majorité, à leur prudence, à leur tact, à leur habileté, qualités le plus souvent citées, ils ont cette attitude qualifiée par l'inspection générale de « rondeur bretonne $^{60}$ " qui leur assure la réussite dans leur académie d'origine.

Lequinquis, originaire de Lannion, entré dans l'inspection en 1838, reste en fonctions en Bretagne, pendant trente-six ans. Alors qu'il est inspecteur des écoles dans le Morbihan, l'inspecteur général le définit de la manière suivante : Modeste, doux mais ferme et connaissant parfaitement toute

54. AN F17/21747 : dossier Jules SpAL, lettre du préfet de Loire-Inférieure au ministre, 29 novembre 1858

55. AN F17/20927 : dossier de Pierre HAMON, rapport de l'inspecteur général Vincent, $1^{\text {er juin } 1857 .}$

56. AN F17/21161 : dossier Joseph LEQUINQUIS, rapport de l'inspecteur général, 1842.

57. AN F17/20514 : dossier Pierre DAMY, rapport du recteur de l'académie de Rennes, 5 août 1860.

58. AN F17/21747 : dossier Jules SpaL, rapport de l'inspecteur général, Jean-Jacques Rapet, octobre 1866.

59. AN F17/20562 : dossier Jean-Marie DeLIVRE, rapport de l'inspecteur général Villemereux, 26 septembre 1859.

60. AN F17/20514 : dossier Damy. Rapport de l'inspecteur Vincent, juin 1857 : " son caractère a toute la rondeur du breton ". 
l'étendue de ses devoirs qu'il remplit d'une manière tout à fait digne d'éloges. Ses rapports sont toujours très bien faits, concluants, péremptoires. C'est un inspecteur véritablement modèle [...]. Toutefois, je ne puis dissimuler que Mr Lequinquis ne se présente pas d'une manière avantageuse au premier aspect. M. Lequinquis n'est pas lettré; il n'a reçu que l'éducation primaire, et pourtant son langage est facile et pur, et ses rapports sont écrits d'une manière très remarquable ${ }^{61}$."

Cette médiation sociale se manifeste également par des initiatives en faveur des couches populaires de la population. Ainsi Eugène Lacôte, inspecteur des arrondissements de Montfort et Redon, en Ille-et-Vilaine, organise, en 1863, une souscription en faveur des ouvriers cantonniers et recueille une somme de plus de $420 \mathrm{~F}^{62}$.

Les inspecteurs sont l'un des seuls corps de fonctionnaires à avoir ce double rôle d'administrateur et de surveillant d'un côté, de pédagogue de l'autre. Ce second aspect du métier, la médiation pédagogique, est enrichi sous le ministère de Gustave Rouland, à partir de $1857^{63}$. Ce sont ces inspecteurs qui poussent les instituteurs à s'abonner aux différents journaux professionnels qui contiennent des leçons modèles et des repères pour l'organisation de la classe (Journal des instituteurs, à partir de 1858). Ils animent des conférences cantonales, guident dans leur enseignement les maîtres de leur circonscription.

Les inspecteurs primaires sont enfin des représentants de l'Université, donc de l'État pendant 20, 30, voire 40 ans, mais doivent également servir le régime en place. Sous la monarchie de Juillet, ils doivent prêter serment au roi, puis à partir de 1848, servir la République. Le serment de fidélité à la Constitution et à l'Empereur est exigé, à partir de 1852, même si, pour certains, cette prestation de serment constitue un déchirement, avoué plus tard ${ }^{64}$. Ils ne doivent pas faire preuve de sentiment anti-républicain sous la III République. Leur accession à l'inspection se fait le plus souvent difficilement, après une longue période d'attente et des demandes réitérées, et jamais sans l'appui d'hommes politiques. Le poids de la politique se lit dans les nombreuses recommandations nécessaires, non seulement pour accéder à la profession, mais également pour changer de circonscription, et passer d'une classe du corps à une autre classe. Sur les documents officiels de candidature, figure une rubrique spécifique : "recommandations".

61. AN F17/21161 : dossier LEQUINQUIS, rapport de l'inspecteur général, 1842.

62. AN F17/21041 : dossier Eugène LACOTE-LAVAUBLOIS, lettre du recteur de l'académie de Rennes au ministre, 27 février 1863.

63. FERRIER, Jean, Les inspecteurs des écoles primaires, op. cit., tome 1, p. 46. Circulaire du 20 août 1857 relative à la direction pédagogique des écoles.

64. Sous la monarchie de Juillet, le serment au roi est prescrit par les lois des 31 août 1830 et 28 juin 1833. Sous le Second Empire, l'inspecteur prête serment, conformément à l'article 14 de la constitution de 1852 et à l'article 3 de l'arrêté du 30 juin 1860 . L'inspecteur prononce la phrase : " Je jure obéissance à la constitution et fidélité à l'Empereur ", puis est officiellement installé dans ses fonctions. 
Pour des fonctionnaires ayant traversé trois ou quatre régimes, ayant prêté serment au roi Louis-Philippe, à l'Empereur Napoléon III, après avoir servi la Seconde République et avant de continuer leur carrière sous la Troisième, il est facile de souligner leur conformisme en politique, voire leur détachement. En Bretagne, les engagements forts en faveur d'un régime sont peu nombreux. Au sein départements de l'Ouest, la Bretagne est moins touchée par les grands moments d'épuration que certains départements voisins, tels la Mayenne ou le Maine-et-Loire. La " rondeur bretonne " a été payante. Sur les 17 inspecteurs en fonctions dans l'académie de Rennes, en 1865, compte non tenu de Lorans, mort dans l'exercice de ses fonctions en 1866, $79 \%$ ont servi dans l'enseignement sous quatre régimes différents et $41 \%$ se sont maintenus dans le corps d'inspection, sans interruption, de Louis-Philippe aux débuts de la Troisième République. Ils sont peu révolutionnaires, voire antirévolutionnaires en 1848, servent l'État impérial et rendent des services aux préfets lors des élections du Second Empire. L'inspecteur Lacôte reçoit les éloges d'Armand Gaultier La Guistière pour son aide lors des élections législatives de $1863^{65}$.

\section{Changements politiques et résistances à l'inspection}

Les affaires politiques concernant les inspecteurs sont peu nombreuses en Bretagne. Certains inspecteurs sont mutés pour avoir approché de trop près la Révolution de 1848. François, Mathurin, Amateur Montier est dans ce cas. Briochin d'origine, inspecteur des écoles dans les Côtes-du-Nord (1844-1847), puis en Ille-et-Vilaine (1847-1849), il est accusé d'avoir fréquenté un comité et le club républicain de Saint-Brieuc en 1849. Il se défend vigoureusement mais ne peut éviter la mutation en Corse, puis dans le Nord. Après un congé de plus de quatre ans, il réintègre l'inspection primaire en 1853, obtenant la circonscription de Beaupréau, dans le Maine-et-Loire. L'épuration du corps consiste surtout à écarter les nouvelles candidatures d'instituteurs du degré supérieur, politiquement peu sûrs, tels François Connen, maîtreadjoint à l'école normale de Rennes, et Moureau, instituteur à Hennebont ${ }^{66}$.

Les années 1870-1871 ne constituent pas une période charnière pour le corps des inspecteurs en général. En revanche, 1877 et les années suivantes, jusqu'en 1880, sont beaucoup plus agitées. Là encore, la Bretagne est peu concernée par la vague de mises à la retraite, de refus de l'honorariat,

65. AN F17/21041 : dossier Eugène LACOTE-LEVAUBLOIS. Lettre du député au Corps législatif, de La Guistière, au ministre de l'Instruction publique, 18-07-1864 : " M. LacôteLevaublois, inspecteur primaire de $3^{\mathrm{e}}$ classe pour les arrondissements de Redon et de Montfort dont je suis le député [...] m'a rendu de grands services, par son habileté et sa modération lors de la lutte qui s'est engagée à l'époque des élections dans notre département, entre les adversaires et les amis de l'Empire ", Éric AncEAU, Dictionnaire des députés du Second Empire, Rennes, PUR, 1999, p. 210. Armand Gaultier La Guistière (1825-1893), député de la quatrième circonscription de l'Ille-et-Vilaine, de 1863 à 1870.

66. Arch. dép. d'Ille-et-Vilaine, 10T4 : Registre du conseil académique de Rennes, 18491854, séance du 4 octobre 1850. 
de mutations-punitions. À l'intérieur de l'académie de Rennes, le contraste est grand entre les deux départements de Mayenne et de Maine-et-Loire où les règlements de compte sont nombreux ${ }^{67}$ et les cinq départements bretons. En Mayenne, l'administration républicaine obtient avec difficulté le départ à la retraite de l'indéracinable inspecteur Gondard qui quitte ses fonctions en 1878, après sept années de résistance.

Dans le Maine-et-Loire, le nouveau préfet Louis Assiot ${ }^{68}$ demande en 1880, non seulement le départ de l'inspecteur d'Académie de son département, mais la mise à la retraite ou le déplacement des quatre inspecteurs primaires du département, à ses yeux tous compromis sous les gouvernements précédents. Au-delà de la volonté de remplacer les anciens inspecteurs ayant servi l'Empire et la Troisième république monarchiste et de les prendre pour boucs émissaires dans un département où les candidats républicains ont du mal à percer, ce sont les arguments utilisés par le préfet qui retiennent l'attention. À deux inspecteurs, Détriché et Michelet, est formulé le reproche, outre d'être hostiles aux institutions et aux personnalités républicaines, d'exercer dans leur département d'origine. Pour le second, s'ajoute le fait d'avoir obtenu la Légion d'honneur en octobre $1877^{69}$.

Dans l'argumentaire du préfet, justifiant un déplacement ou une mise à la retraite des deux autres inspecteurs, figure le lien établi entre l'origine bretonne de ces deux fonctionnaires et le fait qu'ils soient favorables au clergé. « M. Colomb, inspecteur primaire de l'arrondissement de Baugé, originaire de Bretagne, écrit le préfet [...] toujours humble devant le clergé remarquablement impérieux dans ce pays, n'a pas peu contribué à décourager quelques velléités de légitime résistance. " Spal, le dernier inspecteur, cumule les suspicions. Il est né en Loire-Inférieure et est en charge de l'arrondissement de Cholet, ces deux faits suffisant à nourrir chez le nouveau préfet le soupçon contre l'inspecteur, accusé d'entretenir « de nom-

67. Gondard, en Mayenne, dont les autorités académiques réclamaient le changement parvient, en s'appuyant sur son réseau de relations, en particulier auprès du préfet, à conserver son poste jusqu'en 1878.

68. MARAIS, Jean-Luc (dir.), Les préfets de Maine-et-Loire, Rennes, PUR, 2000, p. 54. Marie Louis Félix Auguste Assiot (1834-1893), préfet du département du Maine-et-Loire pendant un an, de janvier 1880 à janvier 1881, se fait rapidement remarquer par sa maladresse et les mesures excessives qu'il prend contre le clergé, contre les maires nobles et contre les fonctionnaires de l'Instruction publique. Les notables républicains demandent son départ neuf mois après sa prise fonctions.

69. AN F17/20601 : dossier personnel de René DetRiche. Dans ce dossier se trouve un rapport concernant son collègue, l'inspecteur primaire Michelet. Ce document a été rédigé à Angers, le 24 mars 1880, par l'inspecteur général Baudouin qui écrit : " Il fut décoré 14 jours avant la chute du 16 mai. Cette décoration qu'il porte longue et large sur tous ses habits, il passe dans l'opinion publique pour ne l'avoir obtenue qu'en récompense de sa complaisance pour la politique du 16 mai. Ce qui est incontestable, c'est que cette décoration lui fut donnée sur les recommandations les plus puissantes de M. de Reinach alors préfet, de trois sénateurs royalistes et des députés les plus réactionnaires du département. " L'inspecteur général ajoute que pendant les six semaines qui ont précédé la chute du 16 mai, il n'a pas résisté au déplacement d'un grand nombre d'instituteurs pour complaire au préfet et qu'il a contresigné sans résistance ces injustes changements. 
breuses attaches dans le camp clérico-légitimiste encore tout puissant dans l'ancienne Vendée militaire ${ }^{70}$ ".

Au sein des cinq départements de la Bretagne historique, seul, en 1873, Hamon est mis à la retraite d'office à la suite d'une plainte de l'évêque de Saint-Brieuc, après 41 ans de service, preuve de la part des différents régimes, monarchique, républicain et impérial qu'une politique prudente a présidé au choix des hommes chargés au niveau subalterne de diriger l'instruction publique au sein de la Bretagne historique. Ceci est également la manifestation de la part du corps d'inspection, d'un véritable attachement au service de l'État, transcendant les changements de régime et les clivages politiques ${ }^{71}$.

Cette adaptabilité des membres de la profession à l'action ambivalente de service de l'État et d'attachement à la région d'origine ne doit pas masquer les obstacles rencontrés et les limites de leur action. Le handicap de la distance géographique est loin d'être négligeable. Les trajets à parcourir sont importants et épuisants pour la santé des personnels. La dépendance budgétaire limite parfois les missions d'inspection. Lorsque les frais de tournées sont épuisés, les inspections s'arrêtent. Les inspecteurs ne sont pas toujours compris de leurs subordonnés, certains instituteurs les trouvant lointains, peu réguliers dans leurs visites. La trop grande stabilité de la fonction dans l'Ouest nuit à l'innovation en matière pédagogique. Dans son rapport sur Rousselot, l'inspecteur de l'arrondissement de Saint-Brieuc en 1866, l'inspecteur général Rapet écrit : «Breton et n'ayant jamais quitté la Bretagne, il n'est pas suffisamment choqué de ce qu'il voit et ne pousse pas assez à la transformation des habitudes ${ }^{72}$. " Les concurrents de l'inspecteur en matière de contrôle scolaire, curés et délégués cantonaux, n'entretiennent pas toujours de bonnes relations avec lui et ne lui fournissent pas toujours les renseignements nécessaires. La résistance à l'épiscopat, défenseur de la liberté des écoles privées est parfois rude. Trois inspecteurs primaires se voient refuser l'entrée d'établissements congréganistes en Ille-et-Vilaine, en Loire Inférieure et dans le Morbihan (externats de filles des Ursulines de Nantes, Montfort, et Châteaugiron, maison des sœurs Hennebont). Il faut une intervention personnelle du ministre Victor Duruy

70. AN F17/20601 : dossier DETRICHE, rapport du préfet du Maine-et-Loire au ministre, Angers, le 24 mars 1880.

71. Arch. dép. d'Ille-et-Vilaine, 11T 25 : affaire de l'inspecteur boulangiste Béziers en 1889. Lettre du commissaire central de la ville de Rennes au préfet, 26 juin 1889; message chiffré du préfet au ministre de l'Intérieur, 31 juillet 1889 : «Permettez-moi de vous confirmer mon télégramme chiffré particulier du 28 juillet et de vous demander d'intervenir instamment auprès de votre collègue de l'Instruction publique pour lui demander le déplacement immédiat de M. Béziers, inspecteur primaire à Rennes qui est à la dévotion de M. Le Bastard, ancien maire révoqué de Rennes, et qui exerce sur le corps des instituteurs une influence très préjudiciable aux intérêts du gouvernement "; message chiffré du Préfet au ministre de l'Intérieur, 5 août 1889 : « Les retards apportés par le ministre de l'Instruction publique au déplacement de M. Béziers [...] dans une autre région pour qu'il ne puisse pas exercer ici son influence sont profondément regrettables. "

72. AN F1721663 : dossier RouSSELOT. Rapport de l'inspecteur général Rapet, juillet 1866. 
pour que, conformément à la loi du 15 mars 1850 et à la circulaire du 27 octobre 1865, les inspections soient partout effectuées.

En dépit de ces réserves, les subordonnées des inspecteurs fournissent une image généralement positive de l'inspection. Lors du concours de 1861, beaucoup d'instituteurs voient dans l'inspecteur une autorité légitime, nécessaire contrepoids " aux caprices des autorités locales " qui enserrent l'instituteur quotidiennement. Ils sont vus comme le symbole du progrès de l'État et de l'école, au détriment de l'Église et des autres surveillants fort critiqués que sont les délégués cantonaux et les curés. Leur présence sur le terrain permet de sortir les maîtres de leur isolement et est ressentie comme un stimulant du travail des maîtres. Leur action est enfin perçue, comme une contribution importante à la formation des maîtres et à l'affirmation d'un véritable esprit de corps chez les instituteurs.

Les inspecteurs qui sont, depuis Guizot, les hommes de l'enseignement primaire sont également, et très majoritairement, des hommes de leur région. Professionnels de l'administration et de l'enseignement, possédant une double vocation administrative et pédagogique, préparés à une double action, dans leur bureau et sur le terrain, dotés d'un double visage tantôt émanation de l'enseignement secondaire, ils sont le plus souvent issus du milieu de leurs subordonnés, les instituteurs. Médiateurs culturels, sociaux et politiques, ils ont, malgré les tensions politiques et la guerre scolaire dans l'Ouest, contribué à pérenniser l'action de l'État en matière d'école primaire, fait progresser la scolarisation de l'Ouest et la laïcisation de l'enseignement primaire par un contrôle et un endiguement des progrès des écoles libres. Ils ont participé à l'affirmation d'un véritable esprit de corps chez les instituteurs, en assurant le lien entre l'administration et la réalité de l'école, en animant des conférences pédagogiques dans les cantons, unité fondamentale de l'école, en participant à la diffusion de la presse professionnelle, en soutenant, voire en présidant des associations corporatistes d'instituteurs. Reconnus comme incontournables par le pouvoir central autant que par leurs subordonnés, les inspecteurs primaires de l'Ouest, serviteurs de l'État ont été les acteurs principaux de ce que Maurice Agulhon appelle " le pouvoir d'État démultiplié ${ }^{73}$ ", sans lequel la politique scolaire des ministres successifs n'aurait pu être appliquée. Majoritairement attachés à leur région, ils ont prouvé, pour reprendre Mona Ozouf, que les chemins de la centralisation et de la francisation de l'administration et de l'école sont plus sinueux et plus complexes qu'on ne l'imagine souvent ${ }^{74}$. Ces inspecteurs ont incarné avant 1880, à leur façon, cette préservation " des petites patries " étudiées par Jean-François Chanet pour la période suivante.

73. AgulHon, Maurice, " Plaidoyer pour les Jacobins. La gauche, l’État et la région dans la tradition historique française ", Le débat, juin 1981, n 13, p. 62, cité par Jean-François CHANET, L'École républicaine et les petites patries, Paris, Aubier, 1996, p. 64.

74. ChAnET, Jean-François, L'École républicaine et les petites patries, Paris, Aubier, 1996, préface de Mona Ozouf, p. 11. 


\section{Annexe : Répartition du temps de carrière (inspecteurs bretons en exercice entre 1840 et 1880)}

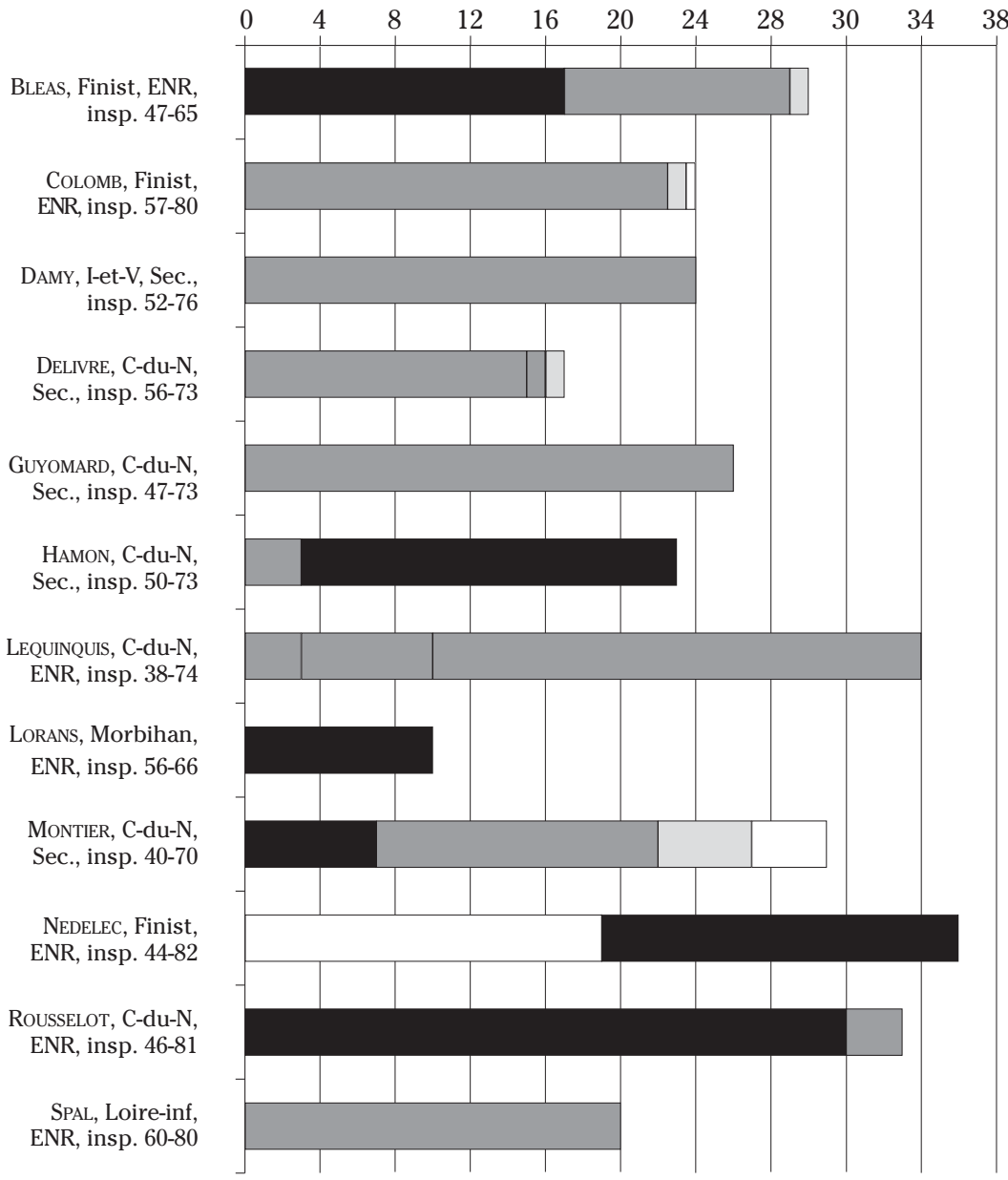

département de naissance

autre département de l'académie

département hors

de l'académie de Rennes

période de congé
ENR : scolarité à l'École normale de Rennes

Sec. : scolarité dans le secondaire

insp. : carrière dans l'inspection primaire (date d'entrée dans le corps - date de la retraite) 


\section{RESUME}

Entre 1835 et 1880, apparaît, puis se développe, le corps d'inspection de l'enseignement primaire, déjà étudié dans le cadre national français, rarement dans celui d'une académie. Fondée principalement sur le dépouillement des dossiers individuels des archives nationales, l'étude s'intéresse aux origines et à la carrière de ces inspecteurs exerçant dans l'académie de Rennes, et essaie d'esquisser la figure-type de l'inspecteur de l'Ouest. Les moyens et les caractères des missions d'inspection, ainsi que les formes de médiation, dans une région marquée par le bilinguisme, les rivalités scolaires et le sousencadrement administratif, constituent un autre volet de la recherche, ouvrant sur la question du compromis entre leurs attaches régionales et le service de l'État, ainsi que sur leur participation à une centralisation prudente, au développement de la scolarisation et à la pérennisation de l'école publique.

\section{ABSTRACT}

Between regional ties and the service of the State : the primary school inspectors in the West in the $19^{\text {th }}$ century

Between 1835 and 1880 appears and develops the corps of primary school inspectors, already studied at the national level in France, rarely at the level of the academy. Based principally on the collection and analysis of individual files of the national archives, the study focuses on the origins and on the careeers of inspectors working in the academy of Rennes and seeks to draw a profile of the typical inspector in the West. The means and the types of inspector assignments, as well as the forms of arbitration, in a region marked by bilingualism, scholastic rivalries, and limited administrative supervision, constitute another aspect of the research which leads to the question of compromise between their regional loyalities and service to the State, as well as to their participation in a judicious centralization, in the development of schooling and the perpetuation of the public school. 